\title{
Risk Factors for Cervical Lymph Node Metastasis of Head and Neck Squamous Cell Carcinoma: A Retrospective Population-Based Study
}

\section{Yamin Zhang}

First Hospital of Zhejiang Province: Zhejiang University School of Medicine First Affiliated Hospital Jing Yu

Zhejiang University School of Medicine First Affiliated Hospital https://orcid.org/0000-0002-1699-7162 Huiyong Zhu ( $\square$ zhycjftt@hotmail.com )

First Hospital of Zhejiang Province: Zhejiang University School of Medicine First Affiliated Hospital

\section{Research}

Keywords: head and neck squamous cell carcinoma, lymph node metastasis, risk factor, tumor size, grade

Posted Date: February 4th, 2022

DOI: https://doi.org/10.21203/rs.3.rs-1203493/v1

License: (a) (1) This work is licensed under a Creative Commons Attribution 4.0 International License. Read Full License

Version of Record: A version of this preprint was published at Journal of Stomatology, Oral and Maxillofacial Surgery on May 6th, 2022. See the published version at https://doi.org/10.1016/j.jormas.2022.05.001. 


\section{Abstract}

\section{Background}

Head and neck squamous cell carcinoma (HNSCC) is characterized by high frequency of early cervical lymph node metastases (LNM), resulting in poor survival of patients. Few researches focused on the risk factors of LNM in HSNCC patients.

\section{Methods}

HNSCC patients diagnosed from 1993 to 2013 in the TCGA database formed the derivation cohort ( $n=316)$; HNSCC patients diagnosed between 2010 and 2020 at the First Affiliated Hospital of Zhejiang University, China, formed the validation cohort $(n=531)$. Clinicopathological data (sex, age, tumor sites, tumor size, and grade) of patients from both cohorts were collected. Their associations with LNM were analyzed using univariate and multivariate logistic regression analysis.

\section{Results}

The tumor size and grade were demonstrated to be independent risk factors for LNM of patients with HNSCC in both the derivation cohort (OR=1.669, 95\% Cl: $1.18 \sim 2.378, \mathrm{p}<0.01$; OR $=1.85,95 \% \mathrm{Cl}$ : 1.287 2.697, $\mathrm{p}<0.01$, respectively) and the validation cohort (OR=1.338, 95\% Cl: 1.015 1.767, $\mathrm{p}<0.05$; $\mathrm{OR}=1.936,95 \% \mathrm{Cl}: 1.46 \sim 2.587, \mathrm{p}<0.0001$, respectively).

Conclusion

Tumors with greater size and higher grade have a greater potential for lymph node metastasis, which remind us the importance of early screening and diagnosis of HNSCC.

\section{Background}

Head and neck squamous cell carcinoma (HNSCC) is the sixth most common malignancy in the world, mainly occurring in the oral cavity, oropharynx, larynx and hypopharynx. ${ }^{1-3}$ Despite the advancement of therapies, the survival rate of HNSCC patients remains poor, with a 5-year survival rate of $65.9 \%$ for the period of 2002-2006. ${ }^{4}$ HNSCCs are generally characterized by local invasiveness and early cervical lymph node metastases (LNM). LNM has been widely accepted as a significant factor influencing the survival and tumor control of HNSCC. ${ }^{5} 6$ Patients with LNM (LNM+) have a poorer 5-year survival rate and higher incidence of distant metastasis than those without LNM (LNM-). ${ }^{7-9}$ Additionally, LNM is an important consideration for surgeons in the determination of surgical plans. Patients clinically diagnosed with LNM ahead of surgery usually undergo neck dissection surgery, which may cause disfigurement, functional impairment, etc. Most of these patients suffer from the subsequent reduction of quality of life. Hence, figuring out the risk factors of LNM is utmost important for patients with HNSCC in surgery decisions on neck dissection. Many researchers have attempted to identify risk factors among various 
clinicopathological features for the LNM of patients with gastric cancer, ${ }^{10,11}$ esophageal cancer, ${ }^{12}$ papillary thyroid cancer, ${ }^{13}$ rectal cancer, ${ }^{14}$ pancreatic cancer,${ }^{15}$ non-small cell lung cancer, ${ }^{16}$ etc. However, few studies, to our knowledge, focused on risk factors of LNM in HNSCC patients.

In addition, many pre-surgery evaluation methods, including palpation, ultrasonography (USG), computed tomography (CT), ${ }^{17}$ fluorodeoxyglucose positron emission tomography/computed tomography (FDGPET/CT), ${ }^{18}$ and sentinel lymph node (SLN) biopsy, ${ }^{19,}{ }^{20}$ have been applied and identified to have various levels of accuracy in the preoperative detection of lymph node metastasis. Unfortunately, these methods still present problems with false positives and false negatives. ${ }^{21-23}$

To prolong survival after curative surgery while avoiding patient over-treatment, more accurate prediction of LNM status are demanded for selecting the strategy of neck section surgery. For the above-mentioned reasons, we aimed to identify the risk factors of LNM in patients with HNSCC.

\section{Methods}

\section{Study design and patients}

We extracted demographic and pathological data of HNSCC patients $(n=501)$ diagnosed between 1993 and 2013 from the TCGA database. The exclusion criteria were as follows: (i) history of previous malignancy; (ii) missing information regarding pathological LNM status; (iii) missing information regarding sex, age, primary tumor region, tumor size, and tumor grade. A total of 139 cases were excluded and 362 eligible cases consist the derivation cohort for further analysis.

An additional independent cohort of primary HNSCC patients was used to validate the role of risk factors for LNM. All patients underwent surgical tumor resection and neck lymph node dissection (elective or therapeutic neck dissection) under general anesthesia at the First Affiliated Hospital of Zhejiang University, China, from January 2010 to June 2020. All specimens were histologically examined by three independent senior pathologists. The exclusion criteria for this cohort were as follows: (i) patients with other malignant tumors; (ii) patients with significant systemic diseases, such as hypertension, diabetes, autoimmune disease and other malignant tumors; (iii) patients that had received chemotherapy, radiotherapy or other biological treatments prior to surgery; (iv) patients with inadequate clinicopathological medical records. A total of 531 patients met the inclusion criteria and were included in the study. The demographic and pathological data of the enrolled patients were obtained from medical records. The collection of retrospective data was approved by the Ethics Committee of the First Affiliated Hospital, College of Medicine, Zhejiang University. The flowchart for patient selection is shown in Figure 1.

\section{Variables}


The survival data (survival time and status) of patients from the derivation cohort was first obtained. Patients were divided into two groups, namely, the group that had no LNM (LNM-) and the group that had LNM (LNM+). The overall survival (OS) of the two groups were compared using the log-rank test.

The variables of patients from both the derivation cohort and the validation cohort, which were included in the univariate and multivariate logistic regression models, were as follows: sex, age at diagnosis, tumor region, $T$ stage, grade, and pathologically lymph node metastasis. All cases were divided into two groups (LNM-, LNM+) based on their pathological LNM status. To minimize the bias and improve the accuracy of analysis results, we further stratified the patients according to the following parameters: (i) sex (male, female); (ii) age ( $\leq 60$ years, $>60$ years); (iii) tumor sites (lip and oral cavity, throat, and overlapping lesion of lip, oral cavity and pharynx); (iv) T stages (T1, T2, T3 T4); and (v) grade (G1, G2, G3 G4). The tumor histology ( $T$ stage and Grade) was categorized according to the TNM staging system of the American Joint Committee on Cancer 7th Edition. ${ }^{24}$

\section{Univariate And Multivariate Regression Analysis}

Variables showing statistically significant effect $(p$-value $<0.05)$ in the univariate analysis were included in the multivariate regression model. Variables with $p$-values less than 0.05 in the multivariate regression model were considered to be independent risk factors for LNM of patients with HNSCC. Before the interpretation of multivariate regression results, the significantly independent variables were assessed for collinearity/multicollinearity using the variance inflation factor (VIF). Values of VIF greater than 1 indicated that variables were collinear; VIF greater than 4 suggested that multicollinearity might be a problem; VIF greater than 10 suggested that multicollinearity might be a serious problem.

\section{Statistical Analyses}

All statistical analyses and plots were performed using GraphPad Prism (version 8.0) and R software (version 4.0.5). The continuous data were expressed as median (range), whereas the categorical variables were described as frequencies and percentages. The survival curves for patients were plotted using the Kaplan-Meier method. The difference between the short-term survival of the two groups was assessed using the Gehan-Breslow-Wilcoxon test, and that between the long-term OS of the two groups was assessed using the log-rank test. Univariate and multivariate logistic regression analysis were used to assess the categorical variables. All statistical tests were two-sided, and a p-value $<0.05$ was considered to be statistically significant.

\section{Results}

\section{Clinicopathological features of HNSCC}


In the derivation cohort, HNSCC patients were predominantly male (73.5\%) and middle-aged (median: 61 years, Q1 Q3: 54 68 years). The median age was 61 years ( $\min \sim \max : 19 \sim 90)$. The oral cavity was the most commonly affected site (55.2\%), followed by the larynx (22.4\%), the overlapping lesion of lip, pharynx (12.7\%), tonsil (5.2\%), pharynx (3.6\%) and lip (8\%). Considering the tumor pathological features, tumors larger than $4 \mathrm{~cm}$ (T3 T4) were the most common (68.5\%), followed by tumors with diameters between 2 and $4 \mathrm{~cm}$ (T2) (24.3\%). Tumors smaller than $2 \mathrm{~cm}(\mathrm{~T} 1)$ were rare $(7.2 \%)$. The majority of tumors were moderately differentiated $(63.2 \%)$, followed by poorly differentiated/undifferentiated $(24.9 \%)$ and well- differentiated (11.9\%) tumors. The incidence of LNM was $57.2 \%$. The demographic and pathological characteristics of these patients, as well as those in the validation cohort, were listed in Table 1. 
Table 1

Demographics and baseline characteristics of patients

\begin{tabular}{|lll|}
\hline Characteristic & Derivation cohort & Validation cohort \\
\hline Age (years) & & \\
\hline Median (min-max) & $61(19-90)$ & $61(25-90)$ \\
\hline$\leq 60$ & $195(53.9 \%)$ & $252(47.5 \%)$ \\
\hline$>60$ & $167(46.1 \%)$ & $279(52.5 \%)$ \\
\hline Sex & & \\
\hline Male & $266(73.5 \%)$ & $359(67.6 \%)$ \\
\hline Female & $96(26.5 \%)$ & $172(32.4 \%)$ \\
\hline Tumor region & & \\
\hline Lip and oral cavity & $203(56.1 \%)$ & $299(56.3 \%)$ \\
\hline Throat & $113(31.2 \%)$ & $170(32 \%)$ \\
\hline Others & $46(12.7 \%)$ & $62(11.7 \%)$ \\
\hline Tumor size (cm) & & \\
\hline$\leq 2$ & $26(7.2 \%)$ & $221(41.6 \%)$ \\
\hline $2-4$ & $88(24.3 \%)$ & $256(48.2 \%)$ \\
\hline$>4$ & $248(68.5 \%)$ & $54(9.6 \%)$ \\
\hline Tumor differentiation degree & $43(11.9 \%)$ & $179(33.7 \%)$ \\
\hline Well & $229(63.2 \%)$ & $288(54.2 \%)$ \\
\hline Moderately & $90(24.9 \%)$ & $64(12.1 \%)$ \\
\hline Poorly/Undifferentiated & & \\
\hline Lymph node metastasis & & \\
\hline Negative & $155(42.8 \%)$ & $(61.4 \%)$ \\
\hline Positive & $207(57.2 \%)$ & \\
\hline Total & & \\
\hline
\end{tabular}

\section{Associations Of Lnm With Overall Survival (Os)}

The overall median OS of $\mathrm{LNM}^{-}$patients was 38.6 months, whereas that of $\mathrm{LNM}^{+}$patients was 37.8 months. The log-rank test showed no significant difference between the overall survival time of the two 
groups (log-rank $\mathrm{p}=0.084 ; \mathrm{HR}=1.272,95 \% \mathrm{Cl}$ : 0.967 1.674) (Figure 1A). However, the Gehan-BreslowWilcoxon test demonstrated a significant difference between the short-term survival of the groups (Wilcoxon p 0.05). Consequently, we compared the 1-year, 3-year and 5-year survival rate of HNSCC patients with or without LNM (Figure 1B D). The results showed that $\mathrm{LNM}^{-}$patients had a significantly higher 3-year survival rate than $\mathrm{LNM}^{+}$patients $(61.2 \%, 50.9 \%$, respectively; log-rank $p<0.05)$. The 1-year and 5-year survival curves of the two groups showed no statistically significant difference (log-rank $\mathrm{p}=0.832 ; \mathrm{HR}=0.955,95 \% \mathrm{Cl}: 0.62 \sim 1.47$; log-rank $\mathrm{p}=0.096 ; \mathrm{HR}=1.273,95 \% \mathrm{Cl}: 0.955 \sim 1.696)$.

\section{Risk Factors For Lnm}

Among variables included in the univariate logistic regression analysis, the tumor size and tumor grade were significantly related to the LNM status of HNSCC. Tumors larger than $2 \mathrm{~cm}$ had $1.607(95 \% \mathrm{Cl}$ : 1.146 2.272; $p<0.01$ ) times the risk of LNM than those smaller than $2 \mathrm{~cm}$. Moderately differentiated and poorly differentiated/undifferentiated tumors had 1.827 times the risk of LNM than the well-differentiated ones (95\% Cl: 1.276 2.65, $\mathrm{p}<0.001)$. Meanwhile, there were no strong associations of age at diagnosis, sex, primary tumor regions with LNM status ( $p>0.05)$. Variables with $p<0.05$ in the univariate logistic regression analysis ( $T$ stage, and grade) were subsequently included in the multivariate logistic regression analysis to determine the independent risk factors of LNM (Table 2). It was shown that both of them were independent risk factors. The tumor differentiation degree contributed more to predicting LNM compared to tumor size $(\mathrm{OR}=1.85,95 \% \mathrm{Cl}$ : 1.287 2.697, $\mathrm{p}<0.01$; $\mathrm{OR}=1.669,95 \% \mathrm{Cl}: 1.18 \sim 2.378, \mathrm{p}<0.01$, respectively). The independent roles of tumor size and grade as risk factors for $L N M$ were also verified in the validation cohort $(\mathrm{OR}=1.936,95 \% \mathrm{Cl}: 1.46 \sim 2.587, \mathrm{p}<0.0001$; $\mathrm{OR}=1.338,95 \% \mathrm{Cl}: 1.015 \sim 1.767, \mathrm{p}<0.05$, respectively) (Table 3 ).

Table 2

Logistic regression analysis of LNM in the derivation cohort $(n=362)$

\begin{tabular}{|lllllll|}
\hline Risk factor & \multicolumn{3}{l}{ Univariate regression analysis } & \multicolumn{3}{c|}{ Multivariate regression analysis } \\
\cline { 2 - 7 } & OR & $95 \% \mathrm{Cl}$ & P-value & OR & $\mathbf{9 5 \%}$ Cl & P-value \\
\hline Gender & 0.713 & $0.445 \sim 1.14$ & 0.157 & - & - & - \\
\hline Age & 0.68 & $0.446 \sim 1.032$ & 0.07 & - & - & - \\
\hline Tumor region & 1.037 & $0.772 \sim 1.395$ & 0.811 & - & - & - \\
\hline T stage & 1.607 & $1.146 \sim 2.272$ & 0.006 & 1.629 & $1.154 \sim 2.314$ & 0.006 \\
Grade & 1.827 & $1.276 \sim 2.65$ & 0.001 & 1.844 & $1.284 \sim 2.685$ & 0.001 \\
\hline
\end{tabular}


Table 3

Logistic regression analysis of LNM in the validation cohort $(n=531)$

\begin{tabular}{|lllllll|}
\hline \multirow{2}{*}{ Risk factor } & \multicolumn{2}{l}{ Univariate regression analysis } & \multicolumn{3}{l|}{ Multivariate regression analysis } \\
\cline { 2 - 7 } & OR & $95 \% \mathrm{Cl}$ & P-value & OR & $95 \% \mathrm{Cl}$ & P-value \\
\hline Gender & 0.916 & $0.628 \sim 1.33$ & 0.647 & - & - & - \\
Age & 1.222 & $0.861 \sim 1.738$ & 0.261 & - & - & - \\
Tumor region & 1.077 & $0.89 \sim 1.319$ & 0.47 & - & - & - \\
T stage & 1.34 & $1.023 \sim 1.759$ & 0.033 & 1.338 & $1.015 \sim 1.767$ & 0.04 \\
Grade & 1.938 & $1.463 \sim 2.585$ & $<0.0001$ & 1.936 & $1.460 \sim 2.587$ & $<0.0001$ \\
\hline
\end{tabular}

\section{Discussion}

Older cancer patients usually have relatively poorer survival rates than younger patients. ${ }^{25,}{ }^{26} \mathrm{HNSCC}$ patients diagnosed at $\geq 60$ years of age have been reported to have increased cause-specific mortality. ${ }^{27}$ As it is known, advanced TNM stage and poor grade are usually associated with the poor survival of cancer patients. However, the association of age with T classification, grade or LNM remains controversial. Some reports stated that the biologic behavior of HNSCC in younger patients was more aggressive compared with that in elderly patients. ${ }^{28-31}$ However, other studies noted no significant difference in T stage or grade between young and old patients with HNSCC. ${ }^{28,32}$ As to the association of LNM with age, previous studies also had inconsistent and controversial findings. A retrospective study based on a large patient cohort of tongue squamous cell carcinoma reported that the LNM rate in young adults (< 40y) was higher than that in middle-age adults (40y 70y). ${ }^{32}$ This conclusion was supported by several other studies. ${ }^{33-35}$ Meanwhile, Jun-Ook et al. noted no significant difference in $\mathrm{N}$ stage between different age groups. ${ }^{31}$ The inconsistency between these findings might be partially due to the differences in population size, inclusion criteria of patients, age range, and age grouping, etc. In this study, we were surprised to find that the LNM rate of young patients ( $<60 y)$ compared to that of old patients $(\geq 60 \mathrm{y})$ showed the opposite tendency in the derivation and the validation cohort. In the derivation cohort, young patients had a slightly but not significantly higher LNM rate compared to old patients $(p=0.0703)$. In contrast, the LNM rate of old patients was higher, though not significantly, than that of young patients $(p=0.261)$ in the validation cohort. The latter may be ascribed to possible patient selection bias in the choice of neck dissection surgery. When formulating the surgical plan, we comprehensively considered the tumor malignancy, the patient's health status, and the patient's expectations of quality of life after surgery. For elderly patients, the choice of neck dissection would be made more rigorously, which might explain the higher LNM rate in our validation cohort than the actual rate in elderly patients with HNSCC. Obviously, more prospective validation research is required to support this assumption. 
As LNM is major prognostic factor for HNSCC patients, figuring out its risk factors is of utmost importance. ${ }^{36,37}$ Increasing evidence indicates that lymphatic metastasis is not entirely passively but also actively regulated by malignant cells. ${ }^{38-40}$ Thus, the characteristics of primary tumors may highly impact the outcome of LNM occurrence. Regarding the associations of LNM with tumor size, we found that greater tumors (T2 T4) had increased the LNM rate 1.607-fold compared to smaller tumors (T1) (95\% Cl:1.146 2.272, $\mathrm{p}<0.05)$. This finding is in good agreement with previous studies. Chung et al. reported that tumors with higher T stage (T3 T4) had higher LNM rate, though with no statistical significance, compared to those with lower T stage (T1) (OR:1.86, 95\% Cl: 0.95 3.77, $p=0.07) .{ }^{41}$ Bataini et al. reported an increased clinical occurrence of LNM in patients with T4 lesions (70\%) compared to those with $\mathrm{T} 1$ lesions (44\%). ${ }^{42}$ Except for T stage, the histologic grading based on malignant histomorphology has been widely used to predict the outcomes of patients with various cancer types, including HNSCC. ${ }^{43-46}$ Kademani, et al. reported that a higher-grade of HNSCC tumors is associated with decreased survival rate. ${ }^{47} \mathrm{~A}$ few studies have proved that tumor grade, especially grading at the invasive front, is a predictor of LNM in HNSCC. ${ }^{48}$ Further reports identified the significant positive correlation of the degree of pathological grade of primary tumors and occurrence frequency of germinal center predominance pattern. ${ }^{49}$ Since HNSCC tumors with germinal center predominance pattern feature twice risk of LNM compared to those with lymphocyte predominance pattern, ${ }^{50,51}$ this may partially explain why high-grade tumors metastasize to regional lymph nodes with higher frequency.

In addition to LNM status, many indicators related to metastatic lymph nodes have been documented to be significant predictors of HNSCC patient prognosis, including the number of metastatic nodes, ${ }^{52}$ lymph node density (number of positive lymph nodes/total number of excised lymph nodes, LND) ${ }^{9,53,54}$ and the presence of extra-nodal spread (ENS) ${ }^{7}$, etc. However, we could not make further associations because of the limitations of the original data.

To the best of our knowledge, this study is one of the first to identify the risk factors for LNM of HNSCC patients from the aspect of tumor pathology. We demonstrate that tumors with greater size and higher grade have a greater potential for lymph node metastasis. Based on this, the early screening and diagnosis of HNSCC is of critical importance for improving treatment efficacy and prolonging overall survival time. Despite its merits, the research has some limitations. Firstly, the present study had a retrospective design and relied upon evaluations of the pathology reports. The heterogeneity of surgeons performing the clinical practice was inevitable. Although patients in both the derivation cohort and the validation cohort received neck dissection, the decisions on procedures made by surgeons varied among individuals. Neck dissection is classified based on the dissected anatomic regions, including radical neck dissection, modified radical neck dissection, selective neck dissection, and extended neck dissection. ${ }^{55}$ The diagnosis of pathological LNM was basically dependent on the performance of neck dissection. The inconsistence of surgical practice might lead to underestimation of the incidence of pathological LNM and of the correlation of LNM with the OS of HNSCC patients, as well as the potential correlations of other variables with LNM. Secondly, the validation cohort was formed by patients from a single 
institution, therefore, a prospective multicenter randomized trial involving a larger number of patients is necessary to validate the findings.

\section{Conclusions}

LNM is associated with poorer 3-year survival rates of patients with HNSCC. Tumor size and tumor differentiation degree are significant risk factors of LNM. Tumors with greater size and higher grade have a greater potential for lymph node metastasis.

\section{Abbreviations}

HNSCC

Head and neck squamous cell carcinoma

LNM

lymph node metastases

USG

ultrasonography

CT

computed tomography

FDG-PET/CT

fluorodeoxyglucose positron emission tomography/computed tomography

SLN

sentinel lymph node

OS

overall survival

VIF

variance inflation factor

OR

odds ratio

$\mathrm{Cl}$

confidence index.

\section{Declarations}

\section{Ethics approval and consent to participate}

The collection of retrospective data was approved by the Ethics Committee of the First Affiliated Hospital, College of Medicine, Zhejiang University.

\section{Consent for publication}


Not applicable.

\section{Availability of data and materials}

The datasets analysed during the current study are available in the TCGA at https://portal.gdc.cancer.gov/. The patients' information at the First Affiliated Hospital of Zhejiang University is available from corresponding author upon reasonable request.

\section{Competing interests}

The authors declare that they have no competing interests.

\section{Funding}

This study was not supported by any specific grant from funding.

\section{Authors' contributions}

YZ: Study concept and design, quality control of data and algorithms, data analysis and interpretation, manuscript preparation; JY: data acquisition, statistical analysis; HZ: manuscript editing and review. All authors have read and approved the final manuscript.

\section{Acknowledgements}

Not applicable.

\section{Authors' information}

${ }^{1}$ Department of Oral and Maxillofacial Surgery, The First Affiliated Hospital of Zhejiang University School of Medicine, Hangzhou, Zhejiang, China; ${ }^{2}$ Zhejiang University School of Medicine, Hangzhou, Zhejiang, China.

\section{References}

1. Pfister DG, Spencer S, Brizel DM, et al. Head and Neck Cancers, Version 1.2015. J Natl Compr Canc Netw. 2015;13:847-55. quiz 56.

2. Gupta B, Johnson NW, Kumar N. Global Epidemiology of Head and Neck Cancers: A Continuing Challenge. Oncology. 2016;91:13-23. 
3. Johnson DE, Burtness B, Leemans CR, Lui VWY, Bauman JE, Grandis JR. Head and neck squamous cell carcinoma. Nat Rev Dis Primers. 2020;6:92.

4. Pulte D, Brenner H. Changes in survival in head and neck cancers in the late 20th and early 21 st century: a period analysis. Oncologist. 2010;15:994-1001.

5. Cerezo L, Millan I, Torre A, Aragon G, Otero J. Prognostic factors for survival and tumor control in cervical lymph node metastases from head and neck cancer. A multivariate study of 492 cases. Cancer. 1992;69:1224-34.

6. Shingaki S, Nomura T, Takada M, Kobayashi T, Suzuki I, Nakajima T. The impact of extranodal spread of lymph node metastases in patients with oral cancer. Int J Oral Maxillofac Surg. 1999;28:279-84.

7. Shingaki S, Takada M, Sasai K, et al. Impact of lymph node metastasis on the pattern of failure and survival in oral carcinomas. Am J Surg. 2003;185:278-84.

8. Shingaki S, Suzuki I, Kobayashi T, Nakajima T. Predicting factors for distant metastases in head and neck carcinomas: an analysis of 103 patients with locoregional control. J Oral Maxillofac Surg. 1996;54:853-7.

9. Patel SG, Amit M, Yen TC, et al. Lymph node density in oral cavity cancer: results of the International Consortium for Outcomes Research. Br J Cancer. 2013;109:2087-95.

10. Jin X, Wu W, Zhao J, et al. Clinical Features and Risk Factors for Lymph Node Metastasis in Early Signet Ring Cell Gastric Cancer. Front Oncol. 2021;11:630675.

11. Du J, Shen Y, Yan W, Wang J. Risk factors of lymph node metastasis in the splenic hilum of gastric cancer patients: a meta-analysis. World J Surg Oncol. 2020;18:233.

12. Wakita A, Motoyama S, Sato Y, et al. Evaluation of metastatic lymph nodes in $\mathrm{CNO}$ thoracic esophageal cancer patients with inconsistent pathological lymph node diagnosis. World J Surg Oncol. 2020;18:111.

13. Zhao H, Huang T, Li H. Risk factors for skip metastasis and lateral lymph node metastasis of papillary thyroid cancer. Surgery. 2019;166:55-60.

14. Ushigome $H$, Ohue $M$, Kitamura $M$, et al. Evaluation of risk factors for lymph node metastasis in $T 2$ lower rectal cancer to perform chemoradiotherapy after local resection. Mol Clin Oncol. 2020;12:390-4.

15. Jiang W, Bai W, Li J, Liu J, Zhao K, Ren L. Leukemia inhibitory factor is a novel biomarker to predict lymph node and distant metastasis in pancreatic cancer. Int J Cancer. 2021;148:1006-13.

16. Moon Y, Choi SY, Park JK, Lee KY. Risk Factors for Occult Lymph Node Metastasis in Peripheral NonSmall Cell Lung Cancer with Invasive Component Size 3 cm or Less. World J Surg. 2020;44:165865.

17. Ishii J, Amagasa T, Tachibana T, Shinozuka K, Shioda S. US and CT evaluation of cervical lymph node metastasis from oral cancer. J Craniomaxillofac Surg. 1991;19:123-7. 
18. Suzuki H, Ogawa T, Terada A, et al. Detection of FDG-PET and FDG-PET/CT in head and neck squamous cell carcinoma. Nihon Jibiinkoka Gakkai Kaiho. 2007;110:629-34.

19. Shoaib T, Soutar DS, MacDonald DG, et al. The accuracy of head and neck carcinoma sentinel lymph node biopsy in the clinically NO neck. Cancer. 2001;91:2077-83.

20. Moya-Plana A, Guerlain J, Casiraghi O, et al. Sentinel lymph node biopsy in head and neck oncology. Bull Cancer. 2020;107:653-9.

21. Khafif RA, Gelbfish GA, Tepper P, Attie JN. Elective radical neck dissection in epidermoid cancer of the head and neck. A retrospective analysis of 853 cases of mouth, pharynx, and larynx cancer. Cancer. 1991;67:67-71.

22. Haberal I, Celik H, Gocmen H, Akmansu H, Yoruk M, Ozeri C. Which is important in the evaluation of metastatic lymph nodes in head and neck cancer: palpation, ultrasonography, or computed tomography? Otolaryngol Head Neck Surg. 2004;130:197-201.

23. Colo AE, Simoes AC, Carvalho AL, et al. Functional microarray analysis suggests repressed cell-cell signaling and cell survival-related modules inhibit progression of head and neck squamous cell carcinoma. BMC Med Genomics. 2011;4:33.

24. Pollaers K, Hinton-Bayre A, Friedland PL, Farah CS. AJCC 8th Edition oral cavity squamous cell carcinoma staging - Is it an improvement on the AJCC 7th Edition? Oral Oncol. 2018;82:23-8.

25. Zeng $H$, Chen $W$, Zheng $R$, et al. Changing cancer survival in China during 2003-15: a pooled analysis of 17 population-based cancer registries. Lancet Glob Health. 2018;6:e555-e67.

26. Lacy PD, Piccirillo JF, Merritt MG, Zequeira MR. Head and neck squamous cell carcinoma: better to be young. Otolaryngol Head Neck Surg. 2000;122:253-8.

27. Farhood Z, Simpson M, Ward GM, Walker RJ, Osazuwa-Peters N. Does anatomic subsite influence oral cavity cancer mortality? A SEER database analysis. Laryngoscope. 2019;129:1400-6.

28. Soudry E, Preis M, Hod R, et al. Squamous cell carcinoma of the oral tongue in patients younger than 30 years: clinicopathologic features and outcome. Clin Otolaryngol. 2010;35:307-12.

29. Sarkaria JN, Harari PM. Oral tongue cancer in young adults less than 40 years of age: rationale for aggressive therapy. Head Neck. 1994;16:107-11.

30. Kourelis K, Tsue T, Girod D, Tawfik O, Sykes K, Shnayder Y. Negative prognostic factors for head and neck cancer in the young. J BUON. 2013;18:459-64.

31. Park JO, Sun DI, Cho KJ, Joo YH, Yoo HJ, Kim MS. Clinical outcome of squamous cell carcinoma of the tongue in young patients: a stage-matched comparative analysis. Clin Exp Otorhinolaryngol. 2010;3:161-5.

32. Oliver JR, Wu SP, Chang CM, et al. Survival of oral tongue squamous cell carcinoma in young adults. Head Neck. 2019;41:2960-8.

33. Zhang YY, Wang DC, Su JZ, Jia LF, Peng X, Yu GY. Clinicopathological characteristics and outcomes of squamous cell carcinoma of the tongue in different age groups. Head Neck. 2017;39:2276-82. 
34. Hilly $\mathrm{O}$, Shkedy $\mathrm{Y}, \mathrm{Hod} \mathrm{R}$, et al. Carcinoma of the oral tongue in patients younger than 30 years: comparison with patients older than 60 years. Oral Oncol. 2013;49:987-90.

35. Funk GF, Karnell LH, Robinson RA, Zhen WK, Trask DK, Hoffman HT. Presentation, treatment, and outcome of oral cavity cancer: a National Cancer Data Base report. Head Neck. 2002;24:165-80.

36. Pereira ER, Jones D, Jung K, Padera TP. The lymph node microenvironment and its role in the progression of metastatic cancer. Semin Cell Dev Biol. 2015;38:98-105.

37. Hamoir M, Schmitz S, Gregoire V. The role of neck dissection in squamous cell carcinoma of the head and neck. Curr Treat Options Oncol. 2014;15:611-24.

38. Podgrabinska S, Skobe M. Role of lymphatic vasculature in regional and distant metastases. Microvasc Res. 2014;95:46-52.

39. Stacker SA, Williams SP, Karnezis T, Shayan R, Fox SB, Achen MG. Lymphangiogenesis and lymphatic vessel remodelling in cancer. Nat Rev Cancer. 2014;14:159-72.

40. Peinado H, Lavotshkin S, Lyden D. The secreted factors responsible for pre-metastatic niche formation: old sayings and new thoughts. Semin Cancer Biol. 2011;21:139-46.

41. Chung MS, Choi YJ, Kim SO, et al. A Scoring System for Prediction of Cervical Lymph Node Metastasis in Patients with Head and Neck Squamous Cell Carcinoma. AJNR Am J Neuroradiol. 2019;40:1049-54.

42. Bataini JP, Bernier J, Brugere J, Jaulerry C, Picco C, Brunin F. Natural history of neck disease in patients with squamous cell carcinoma of oropharynx and pharyngolarynx. Radiother Oncol. 1985;3:245-55.

43. Frierson HF Jr, Cooper PH. Prognostic factors in squamous cell carcinoma of the lower lip. Hum Pathol. 1986;17:346-54.

44. Bekos C, Grimm C, Brodowicz T, et al. Prognostic role of plasma fibrinogen in patients with uterine leiomyosarcoma - a multicenter study. Sci Rep. 2017;7:14474.

45. Liu JF, Chen CY, Chen HT, Chang CS, Tang CH. BL-038, a Benzofuran Derivative, Induces Cell Apoptosis in Human Chondrosarcoma Cells through Reactive Oxygen Species/Mitochondrial Dysfunction and the Caspases Dependent Pathway. Int J Mol Sci. 2016;17.

46. Taghavi N, Yazdi I. Prognostic factors of survival rate in oral squamous cell carcinoma: clinical, histologic, genetic and molecular concepts. Arch Iran Med. 2015;18:314-9.

47. Kademani D, Bell RB, Bagheri S, et al. Prognostic factors in intraoral squamous cell carcinoma: the influence of histologic grade. J Oral Maxillofac Surg. 2005;63:1599-605.

48. Jamadar S, Narayan TV, Shreedhar B, Mohanty L, Shenoy S. Comparative study of various grading systems in oral squamous cell carcinoma and their value in predicting lymph node metastasis. Indian J Dent Res. 2014;25:357-63.

49. Balla H, Uppala D, Majumdar S, Kotina S, Kodati S, Namana M. Evaluation of immunomorphological patterns of cervical lymph nodes in oral squamous cell carcinoma. J Oral Maxillofac Pathol. 2020;24:285-92. 
50. Yadav ST, Madhu Shankari GS, Chatura K, Dhanuja RJ, Rashmi M. Immunomorphological assessment of regional lymph nodes for predicting metastases in oral squamous cell carcinoma. Indian J Dent Res. 2012;23:121-2.

51. Raj LSM, Boaz K, Natarajan S. Prognostic Significance of Lymph Node Pattern in Oral Squamous Cell Carcinoma (OSCC). J Clin Diagn Res. 2014;8:232-5.

52. Mamelle G, Pampurik J, Luboinski B, Lancar R, Lusinchi A, Bosq J. Lymph node prognostic factors in head and neck squamous cell carcinomas. Am J Surg. 1994;168:494-8.

53. Sayed SI, Sharma S, Rane P, et al. Can metastatic lymph node ratio (LNR) predict survival in oral cavity cancer patients? J Surg Oncol. 2013;108:256-63.

54. Rudra S, Spiotto MT, Witt ME, Blair EA, Stenson K, Haraf DJ. Lymph node density--prognostic value in head and neck cancer. Head Neck. 2014;36:266-72.

55. Robbins KT, Medina JE, Wolfe GT, Levine PA, Sessions RB, Pruet CW. Standardizing neck dissection terminology. Official report of the Academy's Committee for Head and Neck Surgery and Oncology. Arch Otolaryngol Head Neck Surg. 1991;117:601-5.

\section{Figures}




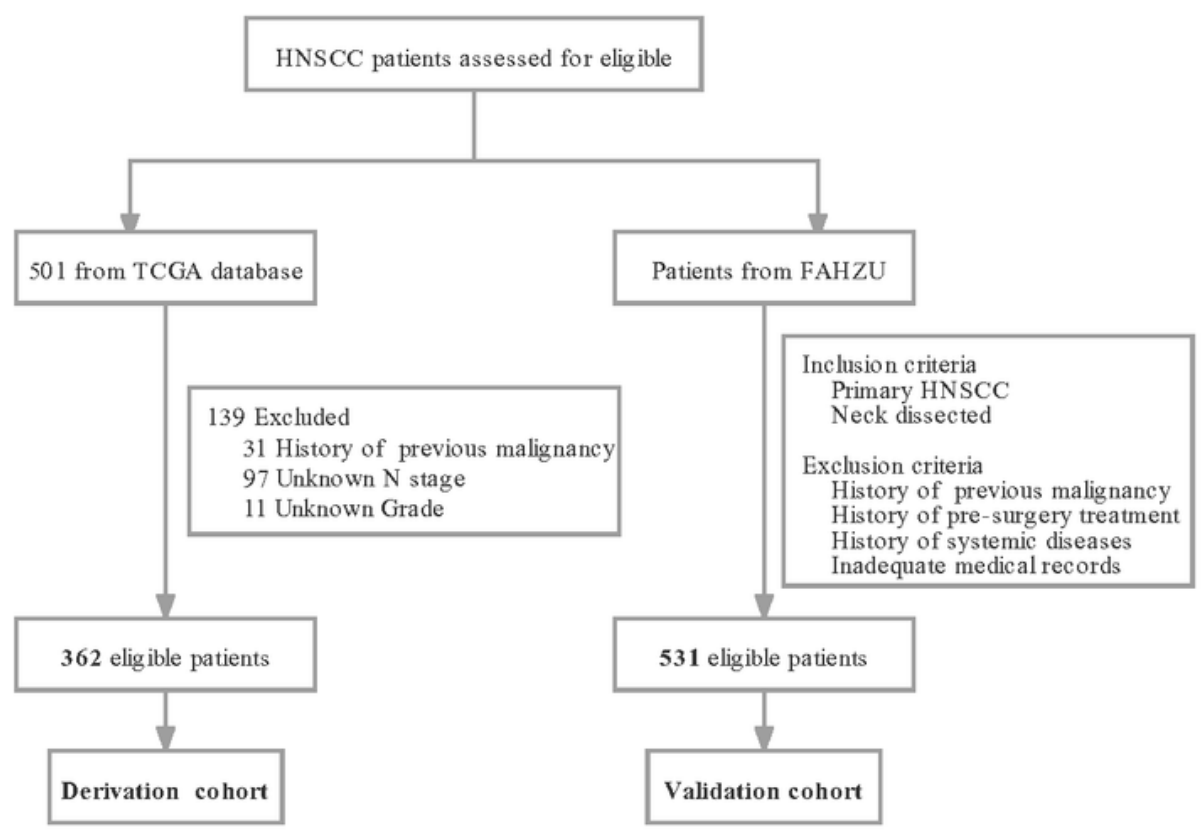

Figure 1

The Flowchart of patient selection in the derivation and validation cohorts. Abbreviations: HNSCC, head and neck squamous cell carcinoma; FAHZU, First Affiliated Hospital of Zhejiang University. 
$\mathbf{A}$

Overall Survival

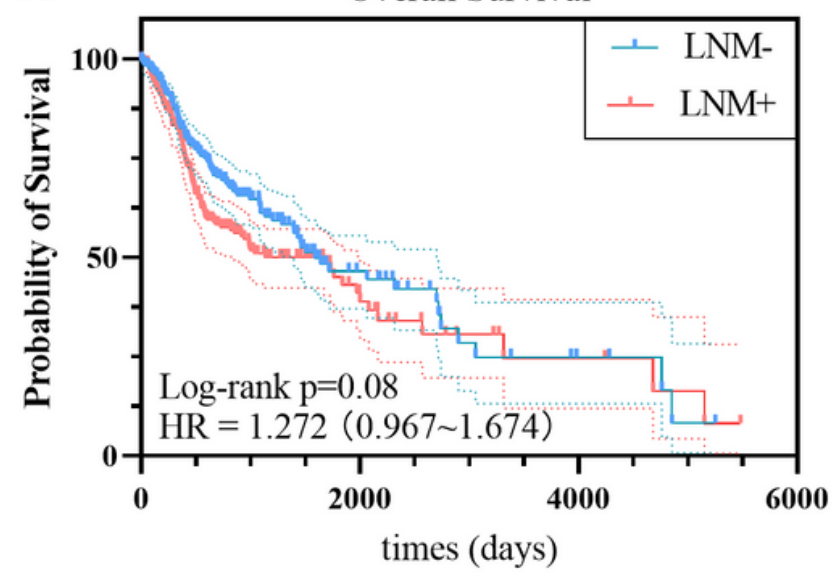

C

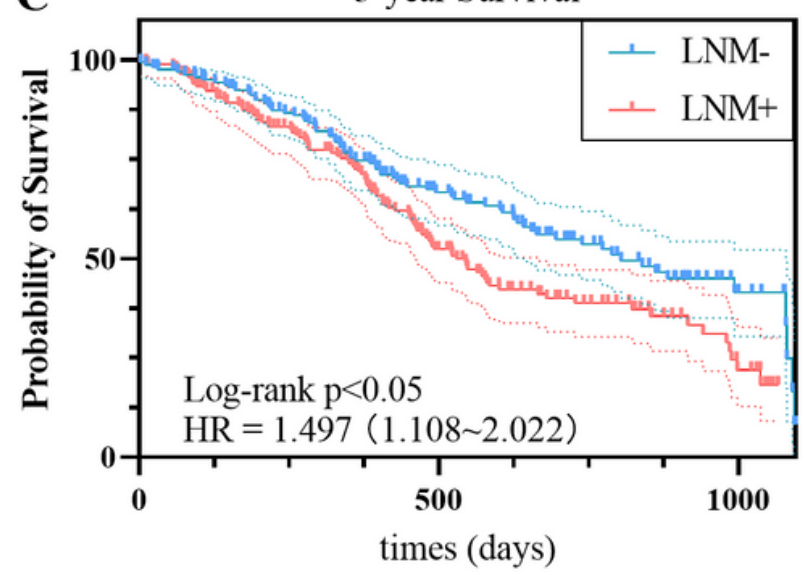

B 1-year Survival

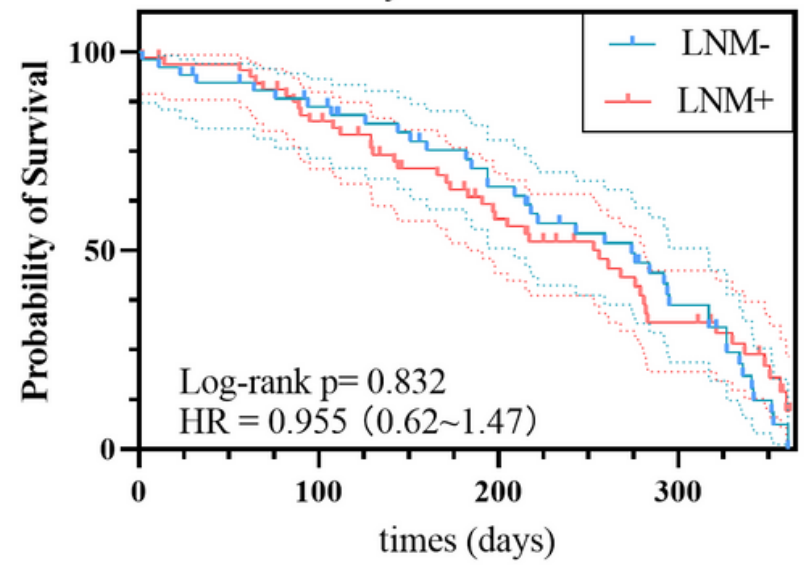

D

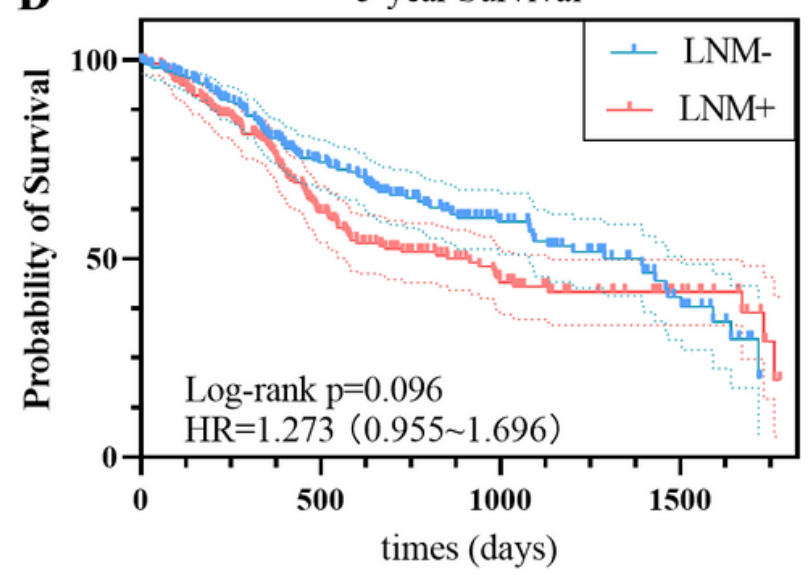

Figure 2

Kaplan-Meier curves of patients with HNSCC, grouped by pathological LNM diagnosis. The red solid and blue solid lines represent HNSCC patients with LNM and those without LNM, respectively. The bands represent pointwise 95\% incidence intervals (Cls). The log-rank P-value, hazard ratio (HR) and corresponding $\mathrm{Cl}$ are shown in each graph. Abbreviations: LNM, lymph node metastasis; $\mathrm{Cl}$, incidence intervals. 